\title{
Nivel de adaptación en el modo desempeño de rol del adulto mayor
}

\author{
Adaptation Level in the Rol Function Mode of the Elderly
}

Iván U. Vilchis-Guerrero ${ }^{a}$, Rubi Ortiz-Godinez ${ }^{b}$, Sergio Rojo-Pérez ${ }^{c}$, Gabriela MaldonadoMuñiz ${ }^{d}$, Benjamín López-Nolasco ${ }^{e}$

\begin{abstract}
:
Introduction: The level of adaptation represents the condition of life processes. Objective: To describe the level of adaptation in the role mode of the older adult in communities of the municipality of Tezontepec de Aldama, Hidalgo. Methodology: quantitative, descriptive study, $\mathrm{n}=195$ older adults whose ages ranged between 60 and 93 years, was developed in 11 communities of the municipality of Tezontepec de Aldama, in the period of June-July 2018, instrument was applied Adaptation level mode role function, validated and with reliability $(\mathrm{KR}-20=0.75)$ and a sociodemographic data questionnaire, informed consent was signed. Results: $64.6 \%$ were female gender $61 \%$ had primary schooling, $56.9 \%$ were married, $65.6 \%$ were homemakers, $81 \%$ received government support, $97 \%$ had social security $20 \%$ lived with diabetes, $24.6 \%$ had hypertension, $12.3 \%$ had diabetes and hypertension, in the adaptation level of the role function mode, $71.3 \%$ were integrated, $28.2 \%$ were compensatory and $0.5 \%$ were committed. Discussion: ChávezRamírez (2017) reports adaptation in the role function mode of $53.7 \%$ high and 46.3 in medium, contrary to that reported in the present study. Conclusions: the level of adaptation of the role function mode of the elderly is integrated.
\end{abstract}

Keywords:

Adaptation, Elderly, Role Function.

\section{Resumen:}

Introducción: El nivel de la adaptación representa la condición de los procesos de vida. Objetivo: Describir el nivel de adaptación en el modo desempeño de rol del adulto mayor en comunidades del municipio de Tezontepec de Aldama, Hidalgo. Metodología: estudio cuantitativo, descriptivo, $\mathrm{n}=195$ adultos mayores cuyas edades oscilaron entre 60 y 93 años, desarrollada en 11 Centros de Salud de comunidades rurales del municipio de Tezontepec de Aldama, en el periodo de Junio-Julio 2018, se aplicó el instrumento Nivel de Adaptación modo desempeño de rol, validado y con confiabilidad (KR-20=0.75), además un cuestionario de datos sociodemográficos, se firmó consentimiento informado. Resultados: $64.6 \%$ eran de género femenino $61 \%$ tenían escolaridad primaria, $56.9 \%$ eran casados, $65.6 \%$ se dedicaban al hogar, $81 \%$ recibían apoyo gubernamental, 97\% contaban con seguridad social, 20\% vivían con diabetes, el $24.6 \%$ con hipertensión, $12.3 \%$ padecían diabetes e hipertensión; en el nivel de adaptación del modo desempeño de rol $71.3 \%$ estuvo integrado, $28.2 \%$ compensatorio y $0.5 \%$ estuvo comprometido. Discusión: Chávez-Ramírez (2017) reporta adaptación en el modo desempeño de rol $53.7 \%$ alto y 46.3 en mediano, contrario a lo reportado en el presente estudio. Conclusiones: el nivel de adaptación del modo desempeño del rol del Adulto mayor se encuentra integrado.

Palabras Clave:

Adaptación, Adulto Mayor, Desempeño de Rol.

a Alumno de la Universidad Autónoma del Estado de Hidalgo, Escuela Superior de Tlahuelilpan. E-mail: vi347872@uaeh.edu.mx

b Alumno de la Universidad Autónoma del Estado de Hidalgo, Escuela Superior de Tlahuelilpan. E-mail: or348571@uaeh.edu.mx

c Alumno de la Universidad Autónoma del Estado de Hidalgo, Escuela Superior de Tlahuelilpan. E-mail: ro346989@uaeh.edu.mx

d Autor de Correspondencia, Profesor Investigador de la Universidad Autónoma del Estado de Hidalgo, Escuela Superior de Tlahuelilpan.

ORCID: https://orcid.org/0000-0002-4967-1812. E-mail: gmaldonado@uaeh.edu.mx

e Profesor Investigador de la Universidad Autónoma del Estado de Hidalgo, Escuela Superior de Tlahuelilpan. ORCID:

https://orcid.org/0000-0003-4566-214X. E-mail: lopez8496@uaeh.edu.mx 


\section{Introducción}

Conforme a las proyecciones del Consejo Nacional de Población (CONAPO), en 2017 residen en el país 12,973 411 personas de 60 y más años $(53.9 \%$ mujeres y $46.1 \%$ hombres). Datos de la Encuesta Nacional de Ingresos y Gastos de los Hogares (ENIGH) 2016, señalan que en el país hay 33.5 millones de hogares de los cuales, en el $30.1 \%$ de estos reside al menos una persona de 60 y más años. De acuerdo al sector laboral, se encontró que $73.2 \%$ trabaja en el sector informal mientras que sólo $37.8 \%$ cuenta con empleo formal; no obstante, en este último rubro se advirtió que el $60.8 \%$ no tiene acceso a instituciones de salud; $61.8 \%$ labora sin tener un contrato escrito y $47.7 \%$ no cuenta con prestaciones laborales. Asimismo, conforme a los datos de la Encuesta Nacional de Empleo y Seguridad Social (ENESS) de 2013, una cuarta parte $(26.1 \%)$ de personas mayores se encuentran pensionadas. De estos, $40.9 \%$ son por jubilación, 33.9\% por retiro o vejez, $17.5 \%$ por viudez y $3.6 \%$ por accidente o enfermedad de trabajo (CNDH, 2017).

El objetivo fue describir el nivel de adaptación en el modo desempeño de rol del adulto mayor en comunidades del municipio de Tezontepec de Aldama, Hidalgo.

\section{Adulto mayor}

La vejez es una de las etapas de la vida: la última, donde el ser humano ha alcanzado su máxima expresión de relación con el mundo; ha llegado a la madurez total a través de una gran cantidad de experiencias adquiridas durante los momentos e instantes disfrutados. Se podría afirmar entonces que la vejez es un subconjunto de fenómenos y procesos que forman parte de un concepto más global: el envejecimiento. Nacemos envejeciendo, vivimos envejeciendo, y así morimos. La vejez es la etapa de la vida en que los síntomas del envejecimiento se hacen más evidentes (Alvarado, 2014).

\section{Adaptación}

La adaptación se refiere al proceso y al resultado por los que las personas, que tienen la capacidad de pensar y de sentir, como individuos o como miembros de un grupo, son conscientes y escogen la integración del ser humano con su entorno. El nivel de la adaptación representa la condición de los procesos de vida. Se describen tres niveles: integrado, compensatorio y comprometido. El nivel de la adaptación afecta la capacidad del sistema humano para responder positivamente en una situación.

El nivel de adaptación es un punto que cambia constantemente, como la interacción entre los humanos y el medio ambiente. Un proceso de vida integrado puede cambiar a compensatorio tratando de provocar la adaptación. Si los procesos compensatorios no son adecuados, resultan los procesos comprometidos (Roy, 2009).

\section{Desempeño de rol}

La categoría del comportamiento que pertenece a los roles en sistemas humanos se llama el modo de desempeño del rol. Este se ocupa específicamente del lugar de la persona en sociedad y cómo actúa en roles recíprocos y roles en grupos. Las metas del modo son integridad y claridad social del rol. Desde la perspectiva del individuo, el modo de desempeño del rol se centra en los papeles que el individuo ocupa en sociedad. Un rol, como la unidad de funcionamiento de la sociedad, se define como sistema de expectativas sobre cómo una persona que ocupa una posición se comporta hacia una persona que ocupa otra posición. La integridad y la claridad social del rol representan las necesidades subyacentes del modo de desempeño del Rol (Roy, 2009).

\section{Planteamiento del problema o exposición del caso}

La adaptación y hacer frente a los cambios del envejecimiento requiere la ayuda de familiares, amigos y profesionales salud, cuando este no tiene una buena adaptación a su vejez, sufre un deterioro psicológico de negación y se debe en su mayoría a la interrupción de la actividad productiva, la separación de los hijos, muerte del - la cónyuge; esto a su vez puede generar tristeza, depresión, melancolía, descuido personal, enojo, ira y todo lo que puede acontecer un rechazo a una etapa fisiológica. Otros indicadores que debemos tener en cuenta para esta investigación son los factores de natalidad, fecundidad y esperanza de vida, ya que estos determinan cuán envejecida está la sociedad desde un punto de vista demográfico. Por lo que, la importancia de estudiar la adaptación en el adulto mayor, deriva del rol de cada uno de ellos. Por tal motivo se plantea la siguiente pregunta: ¿Cuál es el nivel de adaptación del desempeño de rol en el adulto mayor en comunidades del municipio de Tezontepec de Aldama, Hidalgo?

\section{Metodología de diseño o de estudio}

El presente es un estudio es de tipo cuantitativo, por su nivel de alcance exploratorio, aplicada, descriptivo, transversal. Esta investigación se desarrolló en los centros de salud de una comunidad rural de Tezontepec de Aldama, en el periodo de Junio-Julio 2018. Universo conformado por 3,460 adultos mayores 65 años de comunidad de Tezontepec de Aldama, Hidalgo. Muestra calculada mediante la fórmula para proporciones de poblaciones finitas, a lo que nos arrojó la respuesta de $n$ = 192 adultos mayores. Con un muestreo por cuotas, 18 adultos mayores de cada Centro de Salud de las comunidades Tezontepec, Santiago Acayutlan, Atengo, 
Santa Maria Batha, Tenango, Huitel, Presas, Mangas, San Gabriel, Achichilco y La Cruz.

Se aplicó el instrumento nivel de adaptación del desempeño del rol. Consta de 16 ítems, los cuales 15 son en sentido negativo y 1 en positivo; las respuestas son con dos opciones de sí y no. El intervalo es de 0 - 16; las categorías de calificación son tres: comprometido, compensatorio e integrado; así como el cuestionario de datos sociodeográficos, previo firma de Consentimiento Informado.

Se utilizó estadística descriptiva: media, mediana, moda, desviación estándar, frecuencia y porcentaje.

\section{Aplicación}

La enfermería como disciplina práctica, es el conjunto de conocimientos científicos que se utilizan con el propósito de prestar un servicio esencial a las personas, actúa para mejorar la relación entre la persona y el entorno para promover la adaptación. El modelo teórico base para la realización de este trabajo de investigación fue el modelo de adaptación de Callista Roy. Este modelo conceptual plantea unos estímulos que provocan una respuesta, un punto de interacción y relación de la persona con al ambiente.

\section{V.Resultados}

Con la muestra de 195 adultos mayores de la comunidad rural, de la cual el $64.6 \%(n=126)$ eran de género femenino y $35.4 \%(n=69)$ eran de género masculino, de los cuales $61 \%(n=119)$ tenían escolaridad primaria, el $21 \%(n=41)$ tenían secundaria, el $15.4 \%(n=30)$ tenían ninguna, el $21.1 \%(n=4)$ tenían preparatoria y el $0.5 \%(n=1)$ tenían universidad, en el cual su estado civil fue de $56.9 \%$ $(n=111)$ que eran casados, el $31.8 \%(n=62)$ eran viudos, el $7.2 \%(n=14)$ eran solteros y el $4.1 \%(n=8)$ eran divorciados.

La ocupación fue $65.6 \%$ ( $n=128)$ en el hogar, el $14.9 \%$ $(n=29)$ eran jornaleros, el $8.7 \%(n=17)$ eran empleados y el $10.8 \%(n=21)$ eran comerciantes, por lo cual algunos tenían beneficios por parte de gobierno, el cual el $77.4 \%$ $(n=151)$ tenían 65 y más, el $3.6 \%(n=7)$ tenían prospera y el $19 \%(n=37)$ no tenían ninguno. $52.3 \%(n=102)$ vivían con su pareja, el $28.7 \%(n=56)$ con sus hijos, el $17.4 \%$ $(n=34)$ solos y el $1.5 \%(n=3)$ con otros, de los cuales $91.8 \%(n=179)$ tenían casa propia y el $8.2 \%(n=16)$ no.

Con relación a la seguridad social, $80.5 \%(n=157)$ tenía seguro popular, el $8.2 \%$ ( $n=16)$ contaba con IMSS al igual que ISSSTE con un $8.2 \%(n=16)$ y el $3.1 \%(n=6)$ con ninguno, dentro de sus enfermedades se encontraron que el 20\% ( $n=39)$ tenía diabetes, el $24.65 \%(n=48)$ tenían hipertensión, el $12.3 \% \quad(n=24)$ tenían diabetes e hipertensión, solo el $0.5 \%(n=1)$ tenían otras y el $42.6 \%$ ( $n=83$ ) no tenían ninguna enfermedad.

Nivel de adaptación del modo desempeño del rol: $71.3 \%$ $(n=139)$ estuvo integrado, el $28.2 \%(n=55)$ compensatorio y solo el $0.5 \%(n=1)$ estuvo comprometido.

Tabla 1. Frecuencia y porcentaje del nivel de adaptación del modo desempeño de rol.

\begin{tabular}{|l|c|c|}
\hline Nivel de adaptación & $\mathrm{f}$ & $\%$ \\
\hline Integrado & 139 & 71.3 \\
\hline Compensatorio & 55 & 28.2 \\
\hline Comprometido & 1 & .5 \\
\hline
\end{tabular}
de rol.

\section{Discusión}

Chávez Ramirez, en su tesis titulada AfrontamientoAdaptación y la Actividad Social en Adultos Mayores, Arequipa 2017, el estudio fue realizado a 54 adultos mayores de 60 años, menciona que la mayor participación fue del sexo femenino con un $79.6 \%$, cuanto al grado de instrucción predomina el grado superior con un $40.7 \%$, con respecto al estado civil el predominio es casado con un $48.1 \%$, la mayoría tiene como ocupación actual su casa representado por un $55.6 \%$. En el nivel de adaptación en el modo desempeño del rol los valores oscilaron en alto con un $53.7 \%$ alto y 46.3 en mediano; en contraste con los resultados de los adultos mayores de la comunidad rural de Tezontepec, el predominio del sexo femenino en un $64.6 \%$, la escolaridad $61 \%$ tenían primaria, el $21 \%$ secundaria, y $15.4 \%$ ninguna; en el nivel de adaptación el modo desempeño de rol el $71.3 \%$ estuvo integrado, el $28.2 \%$ compensatorio y solo el $0.5 \%$ estuvo comprometido; la diferencia puede ser que Tezontepec es una zona rural por lo tanto su actividad y el desempeño de sus labores es tranquilo mientras que en Arequipa siendo una zona urbana sus labores son más complejas y demandantes en mayor tiempo de ocupación en ellas.

\section{Conclusiones}

El nivel de adaptación del modo desempeño del rol del Adulto mayor es integrado en los centros de salud de la comunidad rural de Tezontepec de Aldama

Debido a las actividades que el adulto mayor realiza en la comunidad rural lo hace estar más activo en su vida diaria llevándolo a integrarse ante las actividades que practica.

\section{Agradecimientos}

A las autoridades del municipio de Tezontepec de Aldama, Hidalgo, México, por su apoyo y a los adultos mayores por su participación. 


\section{Anexos}

\section{Instrumento nivel de adaptación del modo desempeño del rol}

Instrucciones: coloque una $X$ en el cuadro de Sí o NO según corresponda su situación.

\begin{tabular}{|c|c|c|}
\hline PREGUNTA & Sí & No \\
\hline $\begin{array}{l}\text { 1. Le han asignado tareas en su casa } \\
\text { ¿Cuál? }\end{array}$ & 0 & 1 \\
\hline 2. Acepta ayuda de otras personas. & 1 & 0 \\
\hline $\begin{array}{l}\text { 3. Tiene relaciones amistosas fuera del } \\
\text { círculo familiar. }\end{array}$ & 0 & 1 \\
\hline $\begin{array}{l}\text { 4. Realiza actividades adecuadas para } \\
\text { el beneficio de su salud. }\end{array}$ & 0 & 1 \\
\hline $\begin{array}{l}\text { 5. Acepta que hay actividades que ya no } \\
\text { puede realizar. }\end{array}$ & 0 & 1 \\
\hline 6. Se siente preparado para la muerte. & 0 & 1 \\
\hline $\begin{array}{l}\text { 7. Se siente satisfecho con las } \\
\text { actividades que realiza. }\end{array}$ & 0 & 1 \\
\hline $\begin{array}{l}\text { 8. Toma sus medicamentos como se los } \\
\text { indica el médico. }\end{array}$ & 0 & 1 \\
\hline 9. Ingiere la dieta indicada. & 0 & 1 \\
\hline 10. Se baña a diario. & 0 & 1 \\
\hline 11. Se cambia de ropa diario. & 0 & 1 \\
\hline 12. Practica alguna religión. & 0 & 1 \\
\hline 13. Está a gusto con su vida religiosa. & 0 & 1 \\
\hline $\begin{array}{l}\text { 14. Realiza ejercicio físico. } \\
\text { ¿Cuánto tiempo? }\end{array}$ & 0 & 1 \\
\hline 15. Participa en algún equipo deportivo & 0 & 1 \\
\hline 16. Tiene reuniones religiosas & 0 & 1 \\
\hline
\end{tabular}

\section{Referencias}

CNDH. (2017). Emite CNDH informe especial sobre personas mayores en los centros penitenciarios de la República Mexicana. Recuperado el 28 de abril de 2019, de Comisión Nacional de los Derechos Humanos: http://www.cndh.org.mx/sites/all/doc/Comunicados/2017/Co m_2017_318.pdf

Roy C. (2009) The Roy adaptation model. (3a ed.). New Jersey, USA: Pearson Education.

Alvarado-García, A.M. \& Salazar-Maya, Á.M. (2014). Análisis del concepto de envejecimiento. Gerokomos, 25(2).

Recuperado el 28 de junio de 2018:

http://scielo.isciii.es/scielo.php?script=sci_arttext\&pid=S113 4-928X2014000200002 